\title{
Circumvention of Defective Neutral Amino Acid Transport in Hartnup Disease Using Tryptophan Ethyl Ester
}

\author{
Adam J. Jonas and lan J. Butler* \\ Division of Medical Genetics, Department of Pediatrics, Harbor-UCLA Medical Center, Torrance, California 90502; and \\ *Departments of Pediatrics and Neurology, University of Texas Medical School at Houston, Houston, Texas 77030
}

\begin{abstract}
Tryptophan ethyl ester, a lipid-soluble tryptophan derivative, was used to bypass defective gastrointestinal neutral amino acid transport in a child with Hartnup disease. The child's baseline tryptophan concentrations in serum $(20 \pm 6 \mu \mathrm{M})$ and cerebrospinal fluid $(1.0 \pm 0.2 \mu \mathrm{M})$ were persistently $<50 \%$ of normal values. Cerebrospinal fluid 5-hydroxyindoleacetic acid (5-HIAA), a serotonin metabolite, was also $<50 \%$ of normal $(21 \pm 2 \mathrm{ng} / \mathrm{ml})$. Serum tryptophan concentrations increased only modestly and briefly after an oral challenge with 200 $\mathrm{mg} / \mathrm{kg}$ of oral L-tryptophan, reflecting the absorptive defect. An oral challenge with $200 \mathrm{mg} / \mathrm{kg}$ of tryptophan ethyl ester resulted in a prompt increase in serum tryptophan to a peak of $555 \mu$ M. Sustained treatment with $20 \mathrm{mg} / \mathrm{kg} \mathrm{q6h}$ resulted in normalization of serum $(66 \pm 15 \mu \mathrm{M})$ and cerebrospinal fluid tryptophan concentrations (mean $=2.3 \mu \mathrm{M}$ ). Cerebrospinal fluid 5-HIAA increased to more normal concentrations (mean $=33 \mathrm{ng} / \mathrm{ml}$ ). No toxicity was observed over an 8-mo period of treatment, chronic diarrhea resolved, and body weight, which had remained unchanged for 7 mo before ester therapy, increased by $\sim 26 \%$. We concluded that tryptophan ethyl ester is effective at circumventing defective gastrointestinal neutral amino acid transport and may be useful in the treatment of Hartnup disease.
\end{abstract}

\section{Introduction}

Hartnup disorder is an autosomal recessive condition that is characterized by defective renal and gastrointestinal transport of neutral amino acids $(1,2)$. Multiple clinical forms of varying severity have been described (3-5). Many affected individuals appear to be totally asymptomatic (Hartnup disorder), while others suffer from an episodic pellagra-like illness with dermatitis, diarrhea, ataxia, and neuropsychiatric symptoms (Hartnup disease). Infrequently, patients are severely affected in childhood with progressive neurodegeneration leading to death by adolescence. There is little information available concerning the reasons for these different clinical presentations although general nutrition may play a role. It has been suggested that Hartnup disorder is a multifactorial problem, but these factors have not been well identified (6).

Address reprint requests to Dr. Adam J. Jonas, Division of Medical Genetics, Harbor-UCLA Medical Center, 1124 West Carson Street, Torrance, CA 90502.

Received for publication 8 December 1988 and in revised form 22 March 1989.

J. Clin. Invest.

(c) The American Society for Clinical Investigation, Inc.

$0021-9738 / 89 / 07 / 0200 / 05 \$ 2.00$

Volume 84 , July $1989,200-204$
Gastrointestinal absorption of tryptophan, an essential amino acid, is severely impaired in Hartnup disorder and may lead to systemic deficiency of tryptophan and its metabolites, nicotinic acid and NAD. Alternative carriers appear to exist for the uptake of other neutral amino acids that may be present in adequate amounts in serum. Treatment with nicotinamide supplementation to enable NAD synthesis may alleviate some symptoms (7), and a protein enriched diet may also help by providing tryptophan-containing peptide fragments that are absorbed through dipeptide transport systems (8).

Treatment of systemic tryptophan deficiency with nicotinamide may permit synthesis of NAD, but does not directly provide substrate for the synthesis of proteins or serotonin. Under normal circumstances circulating tryptophan enters the central nervous system (CNS) via a transport system for neutral amino acids (9-12). There it is hydroxylated to form 5-hydroxytryptophan and subsequently converted to serotonin by decarboxylation. CNS serotonin concentrations, which are reflected by measurement of the degradation product 5-hydroxyindoleacetic acid (5-HIAA) ${ }^{1}$ in cerebrospinal fluid, are directly related to serum tryptophan concentrations (13-15). Marked increases in brain tryptophan and 5-HIAA concentrations occur within $1 \mathrm{~h}$ after the administration of intravenous tryptophan and are detectable in lumbar cerebrospinal fluid $\sim 6-8 \mathrm{~h}$ later $(12,13)$. While restoration of normal serum tryptophan concentrations would be desireable in Hartnup disorder, this has not been readily achieved. Previous studies demonstrated that amino acid esters readily cross membranes due to their lipid-soluble characteristics and are subject to intracellular enzymatic hydrolysis, thus regenerating the native amino acid (16-19). We investigated tryptophan ethyl ester as a means of bypassing defective amino acid transport in a severely affected child with Hartnup disease whose CNS tryptophan and serotonin levels remained low despite nicotinamide therapy.

\section{Methods}

Study patient. A 3-yr-old child was evaluated for growth failure, marked developmental delay, hyperactivity, chronic diarrhea, episodic ataxia, and weakness. Dietary history was unremarkable and family history was unobtainable. At $3 \mathrm{yr}$ of age height was $87.5 \mathrm{~cm}$ and weight was $10.5 \mathrm{~kg}$, both less than the third percentile for age. His extrapolated Bayley mental development index was 50 and magnetic resonance imaging revealed cortical atrophy. Muscle biopsy of the vastus lateralis showed a nonspecific pattern of type II fiber atrophy. He was noted to have an aminoaciduria that was specific for neutral amino acids with the exception of glycine and imino acids consistent with the pattern seen in Hartnup disease. A D-xylose absorption test was normal.

1. Abbreviations used in this paper: CSF, cerebrospinal fluid; 5-HIAA, 5-hydroxyindoleacetic acid; HVA, homovanillic acid; MHPG, 3methoxy-4-hydroxyphenylethylene glycol. 
Protocols. Informed consent for the following studies was obtained from the child's parents under protocols that had been approved by the local institutional review board. Informed consent for oral tryptophan loading was also obtained from an unrelated adult control.

Oral tryptophan loading. L-Tryptophan was administered orally in a dose of $200 \mathrm{mg} / \mathrm{kg}$ to both the patient and an unrelated normal control. Tryptophan was measured in serum samples using a fluorometric method (20), while other amino acids were measured by automated amino acid analysis (21). Urine indicans were measured spectrophotometrically (22).

Dietary therapy. A diet containing $3 \mathrm{~g} / \mathrm{kg}$ of protein was instituted and maintained throughout the study. Oral nicotinamide supplementation was started at a daily dose of $2 \mathrm{mg} / \mathrm{kg}$ and increased monthly by $2 \mathrm{mg} / \mathrm{kg}$ until a total dose of $10 \mathrm{mg} / \mathrm{kg}$ (given in two divided doses) was achieved. Serum and cerebrospinal fluid (CSF) samples were obtained 4 wk after each increase in nicotinamide supplementation. Tryptophan-derived 5-HIAA, tyrosine-derived 3-methoxy-4-hydroxyphenylethylene glycol (MHPG), and homovanillic acid (HVA) were measured in CSF by gas chromatography and mass spectrometry (23). NAD levels in red cells were measured spectrophotometrically (24).

Intravenous tryptophan loading. Intravenous grade, pyrogen-free L-tryptophan (Ajinomoto USA, Inc., Raleigh, NC) was prepared just before use as a $1 \%$ solution (wt/vol) in 0.5 normal saline, adjusted to pH 7.4 with $1 \mathrm{~N}$ sodium hydroxide, and sterilized by filtration through a $0.22-\mu \mathrm{m}$ filter. After an overnight fast tryptophan was administered intravenously over $30 \mathrm{~min}$ at a dose of $100 \mathrm{mg} / \mathrm{kg}$. Serum samples were obtained for tryptophan measurement over a 6-h period. CSF for tryptophan and serotonin metabolite studies was obtained both before and $8 \mathrm{~h}$ after infusion.

Oral tryptophan ethyl ester loading. After an overnight fast, L-tryptophan ethyl ester (Sigma Chemical Co., St. Louis, MO) was administered as a $2 \%$ solution $(w t / v o l)$ in water by nasogastric tube. Serum samples were obtained over a 6-h period. Tryptophan ethyl ester levels were measured by both HPLC using a $\mathrm{C} 18$ bondapack column and methanol solvent with detection at $280 \mathrm{~nm}$ and by gas chromatography with mass spectrometry. Serum ethanol levels were measured using a NAD-generating system (25).

Tryptophan ethyl ester therapy. The patient was maintained on a protein enriched diet without nicotinamide supplementation. Tryptophan ethyl ester ( $1.25 \%$ solution $\mathrm{wt} / \mathrm{vol})$ was administered orally at a dose of $20 \mathrm{mg} / \mathrm{kg} \mathrm{q6h}$. This dose was chosen to elevate serum tryptophan levels to within the normal range.

Synthesis of tryptophan ethyl ester. L- $\left[{ }^{3} \mathrm{H}\right]$ Tryptophan (ICN Nutritional Biochemicals, Cleveland, $\mathrm{OH}$ ) was esterified using ethanolic $\mathrm{HCl}(20)$. The product was $\sim 98 \%$ pure as determined by TLC on cellulose acetate plates using a solvent system of $96 \%$ methanol/3.7\% $\mathrm{H}_{2} \mathrm{O} / 0.3 \%$ formic acid (26).

In vitro studies. Female Sprague-Dawley rats were anesthetized with $\mathrm{CO}_{2}$ and killed by decapitation. Tissues were removed and homogenized in PBS, pH 7.4, at $4^{\circ} \mathrm{C}$ using a Dounce homogenizer (Kontes Glass Co., Vineland, NJ). Small intestine was flushed extensively with buffer before the mucosa was removed by scraping with a glass slide. Incubations were performed at $37^{\circ} \mathrm{C}$ with $10 \mathrm{mM} \mathrm{L}-\left[{ }^{3} \mathrm{H}\right]-$ tryptophan ethyl ester and then terminated by the addition of $4 \mathrm{vol}$ cold ethanol. After centrifugation for $2 \mathrm{~min}$ at $10,000 \mathrm{~g}$ supernatants were assayed by TLC. Protein was determined spectrophotometrically (27).

\section{Results}

Oral tryptophan and dietary therapy. Challenge with 200 $\mathrm{mg} / \mathrm{kg}$ of oral L-tryptophan resulted in a small increase in serum tryptophan concentration (Fig. 1) with marked indicanuria 16-24 h later, confirming the diagnosis of Hartnup disease. In contrast, serum levels rose to $\sim 600 \mu \mathrm{M}$ in the normal control without marked indicanuria (data not shown). Treatment with a protein enriched diet and oral nicotinamide

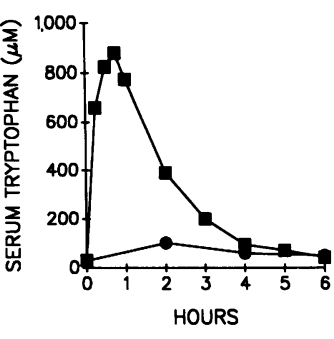

resulted in little or no clinical improvement despite nicotinamide doses as great as $10 \mathrm{mg} / \mathrm{kg}$ per $\mathrm{d}$. Red cell NAD levels on this therapy were $127 \pm 6 \mathrm{nmol} / \mathrm{ml}$ packed red blood cells vs. $81 \pm 7 \mathrm{nmol} / \mathrm{ml}$ for normal controls and literature normal values of $79 \pm 9 \mathrm{nmol} / \mathrm{ml}(24)$. Tryptophan concentrations in serum were approximately one-third of normal at $20 \pm 6 \mu \mathrm{M}$ vs. $62 \pm 21$ for age-matched controls. CSF tryptophan and 5-HIAA concentrations were consistently $<50 \%$ of normal for age (Table I). During a 12-mo period of treatment with nicotinamide his symptoms continued and he had poor growth. His weight reached a peak of $11.6 \mathrm{~kg}$ at $4 \mathrm{yr}$ of age and remained unchanged over the following $7 \mathrm{mo}$.

Intravenous tryptophan. Intravenous tryptophan was administered to test his ability to synthesize serotonin in the CNS. Serum tryptophan concentrations rose promptly after intravenous loading with a peak of $880 \mu \mathrm{M}$ at $45 \mathrm{~min}$ (Fig. 1). $8 \mathrm{~h}$ after infusion the CSF concentration of tryptophan rose sevenfold and the level of 5-HIAA in CSF increased to 50 $\mathrm{ng} / \mathrm{ml}$, within the range of normal (Table I). Transient, mild sedation was the only observed side effect during the study.

Oral tryptophan ethyl ester trial. The lipid-soluble ethyl ester derivative of tryptophan was administered in an effort to bypass the defect in tryptophan absorption. In vitro studies showed that tryptophan ethyl ester is readily hydrolyzed to tryptophan by homogenates of rat intestinal mucosa, liver, and kidney, but not brain (Fig. 2). Virtually no hydrolysis was observed using human plasma or washed human red cells (data not shown). The drug was found to be stable in acidic aqueous solution while refrigerated and protected from light. Over a 5-mo period the purity of a $2 \%$ (wt/vol) solution in 100 $\mathrm{mM}$ citrate buffer, $\mathrm{pH} 4.0$, decreased slightly from 98 to $95 \%$.

After a $200 \mathrm{mg} / \mathrm{kg}$ oral dose the child's serum tryptophan concentration rose to a peak of $555 \mu \mathrm{M}$ and remained elevated

Table I. CSF Metabolites during Tryptophan Ethyl Ester Therapy

\begin{tabular}{lcccc}
\hline \multicolumn{1}{c}{ Treatment } & Tryptophan & 5-HIAA & MHPG & HVA \\
\hline & $\mu M$ & $n g / m l$ & $n g / m l$ & $n g / m l$ \\
& & & $11 \pm 1$ & $95 \pm 31$ \\
Nicotinamide & $1.0 \pm 0.2$ & $21 \pm 2$ & 11 & 9 \\
Intravenous tryptophan & 7.0 & 50 & 9 & 115 \\
Oral ester & $3.3 \pm 1.4$ & $34 \pm 5$ & $11 \pm 3$ & $63 \pm 24^{*}$ \\
Controls & $2.5 \pm 1.1$ & $52 \pm 15$ & $13 \pm 3$ & $103 \pm 29$
\end{tabular}

CSF was obtained by lumbar puncture $7 \mathrm{~h}$ after intravenous tryptophan infusion and after treatment with $20 \mathrm{mg} / \mathrm{kg}$ q6h of tryptophan ethyl ester. Control values were established using CSF from children ages 3-4 yr who had spinal taps performed to rule out infection. * HVA value includes one extremely low value $(35 \mathrm{ng} / \mathrm{ml})$ after which the dose of ester was decreased. 


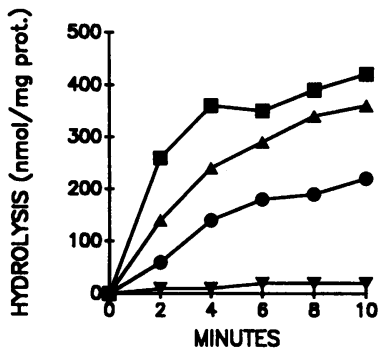

Figure 2. Hydrolysis of $10 \mathrm{mM} \mathrm{L-}$ $\left[{ }^{3} \mathrm{H}\right]$ tryptophan ethyl ester in PBS, pH 7.4, at $37^{\circ} \mathrm{C}$ by homogenates of rat intestinal mucosa (๑), liver $(\boldsymbol{\Lambda})$, kidney ( () , and brain $(\boldsymbol{\nabla})$.

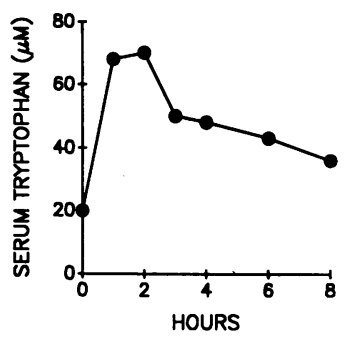

Figure 4. Serum tryptophan levels after a $20 \mathrm{mg} / \mathrm{kg}$ dose of oral L-tryptophan ethyl ester. for $5 \mathrm{~h}$ (Fig. 3). Hydrolysis of the ester during absorption was apparently complete since only tryptophan was detected in serum samples by both HPLC and gas chromatography with mass spectrometry. The lower limit of detection for the ester in serum was $400 \mathrm{nM}$ by the latter method. Ethanol generated by ester hydrolysis was below measurable limits in serum samples as well. Side effects were limited to transient nausea and sedation similar to what has been reported with intravenous tryptophan loading (28). No changes were observed in hematocrit, white count, electrolytes, calcium, phosphorus, or indices of hepatic and renal function $24 \mathrm{~h}$ after the study.

Tryptophan ethyl ester therapy. The dose of tryptophan ethyl ester was adjusted to $20 \mathrm{mg} / \mathrm{kg}$ and was repeated every 6 h based on kinetic data (Fig. 4). Peak serum tryptophan concentrations, measured $1 \mathrm{~h}$ after a dose, were reproducible at $78 \pm 17 \mu \mathrm{M}$, mean $\pm \mathrm{SD}, n=5$ (normal, 62 $n 21 \mu \mathrm{M} ; n=11$ ) over a 1-wk period. No significant changes were observed in the serum concentrations of other amino acids after each dose. Red cell NAD levels were normal at $91 \pm 6 \mathrm{nmol} / \mathrm{ml}$ packed red blood cells. The urinary excretion of tryptophan increased fourfold (from 0.28 to $1.07 \mathrm{mmol} / \mathrm{g}$ creatinine) on this treatment, while the excretion of other amino acids remained unchanged. Urine concentrations of indoleacetic acid as measured by gas chromatography and mass spectrometry remained unchanged by treatment. During therapy the CSF tryptophan concentration approximately tripled over basal levels to $3.3 \pm 1.4 \mu \mathrm{M}$, while concentrations of other amino acids remained in the normal range. The concentration of 5 -HIAA also increased from $21 \pm 2 \mathrm{ng} / \mathrm{ml}$ to $34 \pm 5 \mathrm{ng} / \mathrm{ml}$, while concentrations of MHPG and HVA, tyrosine-derived neurotransmitter metabolites, remained essentially unchanged and within the normal range (Table I). The dose of ethyl ester was decreased by $25 \%$ to $20 \mathrm{mg} / \mathrm{kg}$ three times daily after $5 \mathrm{mo}$ because the CSF tryptophan concentration increased to $5 \mu \mathrm{M}$ and the concentration of HVA markedly decreased to 37 $\mathrm{ng} / \mathrm{ml}$. Study 2 mo later showed normal CSF tryptophan and neurotransmitter concentrations.

His weight, which had been unchanged at $11.6 \mathrm{~kg}$ for the preceding $7 \mathrm{mo}$, increased by $26 \%$ during $8 \mathrm{mo}$ of treatment with tryptophan ethyl ester (Fig. 5). During this same time his linear growth rate doubled as his height increased from 98 to $103 \mathrm{~cm}$. His chronic diarrhea completely resolved and his gait improved. Peak tryptophan concentrations remained normal during treatment at $66 \pm 15 \mu \mathrm{M}, n=4$. Serum concentrations of many essential and nonessential amino acids which were either below or at the lower limit of the normal range before the administration of tryptophan ethyl ester increased (Table II). No side effects were observed other than a faint smell of alcohol on his breath associated with drug ingestion. No changes were observed in either blood counts, serum chemistries, renal function, or hepatic function. Aminoaciduria in spot urine specimens remained unchanged despite therapy.

\section{Discussion}

This patient, with clinical and biochemical criteria diagnostic of Hartnup disease, was unimproved after therapy with nicotinamide and a protein enriched diet. Clinical symptoms including diarrhea, neurologic dysfunction, and poor growth persisted. Serum concentrations of many amino acids, particularly tryptophan, remained low, while concentrations of tryptophan and its metabolites in CSF were also markedly decreased. It has been suggested, but not proven, that tryptophan deficiency is the basis for symptoms in this disorder. Since both transport of tryptophan across the blood-brain barrier and subsequent serotonin synthesis by the CNS appeared to be intact, we directed our efforts toward restoration of normal serum tryptophan concentrations.

We considered tryptophan ethyl ester as a means of bypassing defective gastrointestinal transport of tryptophan in Hartnup disease in light of studies in which lipid-soluble amino acid esters had been used successfully to bypass defective lysosomal transport systems (16-19). The theoretical attractiveness of this approach was strengthened by in vitro experiments that demonstrated that the ester was hydrolyzed by intestinal mucosa, liver, and kidney to provide tryptophan (Fig. 6). As suggested by these studies, tryptophan ethyl ester administration successfully corrected our patient's tryptophan deficiency state. Serum and CSF tryptophan concentrations

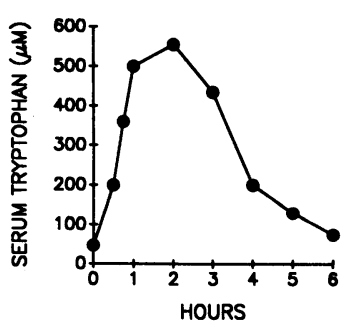

Figure 3. Serum tryptophan levels after a $200 \mathrm{mg} / \mathrm{kg}$ dose of oral L-tryptophan ethyl ester. Baseline serum tryptophan concentration was $35 \mu \mathrm{M}$ at the start of the study.

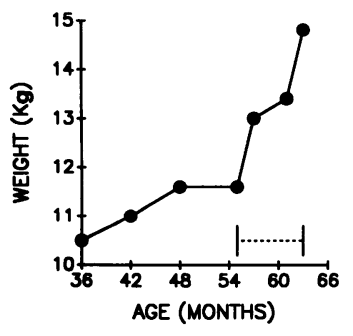

Figure 5. Weight gain over a period of $27 \mathrm{mo}$. Dashed line, interval of therapy with L-tryptophan ethyl ester. 
Table II. Serum Amino Acid Measurements

\begin{tabular}{lcccc}
\hline \multicolumn{1}{c}{ Amino acid } & Normal & Pretherapy & Posttherapy & Ratio \\
\hline & $\mu M$ & $\mu M$ & $\mu M$ & \\
Taurine & $72 \pm 44$ & 24 & 69 & 2.9 \\
Aspartate & $14 \pm 12$ & 5 & 25 & 5.0 \\
Threonine* & $147 \pm 124$ & 37 & 70 & 1.9 \\
Serine & $141 \pm 59$ & 58 & 109 & 1.8 \\
Glu/gln & $561 \pm 220$ & 359 & 373 & 1.0 \\
Proline & $226 \pm 147$ & 143 & 282 & 2.0 \\
Glycine & $244 \pm 60$ & 135 & 152 & 1.1 \\
Alanine & $407 \pm 175$ & 159 & 157 & 1.0 \\
Valine* & $202 \pm 102$ & 97 & 102 & 1.1 \\
Cystine & $44 \pm 16$ & 13 & 23 & 1.8 \\
Methionine* & $52 \pm 35$ & 9 & 67 & 7.4 \\
Isoleucine* & $58 \pm 34$ & 35 & 71 & 2.0 \\
Leucine* & $99 \pm 55$ & 55 & 137 & 2.5 \\
Tyrosine & $167 \pm 170$ & 40 & 34 & 0.9 \\
Phenylalanine* & $79 \pm 46$ & 21 & 32 & 1.5 \\
Ornithine & $138 \pm 133$ & 14 & 105 & 7.5 \\
Lysine* & $173 \pm 100$ & 53 & 146 & 2.8 \\
Histidine* & $78 \pm 24$ & 10 & 31 & 3.1 \\
Arginine* & $83 \pm 54$ & 10 & 66 & 6.6 \\
& & & & \\
\hline & & & &
\end{tabular}

Measurements were performed after an overnight fast both before and after 3 mo of therapy with tryptophan ethyl ester. Normal values are based on data from 20 healthy age-matched controls and are represented by mean $\pm 1 \mathrm{SD}$.

* Essential amino acids.

increased with the subsequent synthesis of serotonin in the CNS. Interestingly, his diarrhea resolved and he had a substantial improvement in growth.

The similarity between symptoms in some individuals with Hartnup disease and those with the niacin deficiency state, pellagra, has focused attention on tryptophan deficiency as a critical etiologic factor. While isolated tryptophan deficiency may lead to chronic neurotransmitter imbalance and theoretically could limit protein synthesis and therefore growth, deficiencies of many other amino acids may also occur in Hartnup disorder (8). In our patient some of these deficiencies may have been aggravated by chronic diarrhea since many, although not all, abnormalities in serum amino acid concentrations resolved with the cessation of diarrhea. The clinical and biochemical improvements observed in our patient in response to tryptophan replacement are suggestive that tryptophan deficiency was a crucial factor in the appearance of his symptoms. Since tryptophan ethyl ester is a specific means of providing tryptophan to these patients, its use in clinical stud-

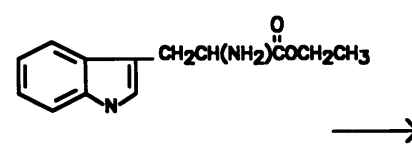

TRYPTOPHW ETHM ESTER

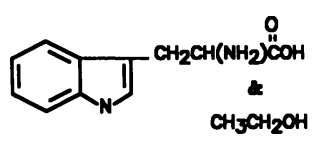

TRYPTOPHW

ETHWOL

Figure 6. Hydrolysis of tryptophan ethyl ester.

ies should now permit the role of tryptophan deficiency in Hartnup disease to be clearly defined.

\section{Acknowledgments}

The authors would like to thank Claire Brunson, Christine Caprioli, Dr. Julie E. Noble, and Dr. Julane Hotchkiss for their invaluable aid.

This work was supported by National Institutes of Health grants 1MO1RR-02558 and DK-37403.

\section{References}

1. Milne, M. D., M. A. Crawford, C. B. Girao, and L. W. Loughridge. 1960. The metabolic disorder in Hartnup disease. $Q . J$. Med. 29:407-421.

2. Scriver, C. S. 1965. Hartnup disease. N. Engl. J. Med. 273:530532.

3. Pomeroy, J., M. L. Efron, J. Dayman, and D. Hoefnagal. 1968. Hartnup disorder in a New England family. N. Engl. J. Med. 278:1214-1216.

4. Tahmoush, A. J., D. H. Alpers, R. D. Feigin, V. Armbrustmacher, and A. L. Prensky. 1976. Hartnup disease. Arch. Neurol. 33:797-807.

5. Wilcken, B., J. S. Yu, and D. A. Brown. 1977. Natural history of Hartnup disease. Arch. Dis. Child. 52:38-40.

6. Scriver, C. R., B. Mahon, H. L. Levy, C. L. Clow, T. M. Reade, J. Kronick, B. Lemieux, and C. Laberge. 1987. The Hartnup phenotype: Mendelian transport disorder, multifactorial disease. Am. J. Hum. Genet. 40:401-412.

7. Wong, P. W. K., A. M. Lambert, P. M. Pillai, and P. M. Jones. 1967. Observations on nicotinic acid therapy in Hartnup disease. Arch. Dis. Child. 42:642-646.

8. Asatoor, A. M., B. Cheng, K. D. Edwards, A. F. Lant, D. M. Matthews, M. D. Milne, F. Navah, and A. J. Richards. 1970. Intestinal absorption of two dipeptides in Hartnup disease. Gut. 11:380-387.

9. Daniel, P. M., S. R. Moorhouse, and O. E. Pratt. 1976. Amino acid precursors of monoamine neurotransmitters and some factors influencing their supply to the brain. Psychol. Med. 6:277-286.

10. Olendorf, W. H. 1973. Saturation of blood brain barrier transport of amino acid in phenylketonuria. Arch. Neurol. 28:45-48.

11. Fernstrom, J. D., and R. J. Wurtman. 1972. Brain serotonin content: physiological regulation by plasma neutral amino acids. Science (Wash. DC). 178:414-416.

12. Fernstrom, J. D., and R. J. Wurtman. 1971. Brain serotonin content: physiological dependence on plasma tryptophan levels. Science (Wash. DC). 173:149-152.

13. Eccleston, D., G. W. Ashcroft, T. B. B. Crawford, J. B. Stanton, D. Wood, and P. H. McTurk. 1970. Effect of tryptophan administration of 5HIAA in cerebrospinal fluid in man. J. Neurol. Neurosurg. Psychiatry. 33:269-272.

14. Modigh, K. 1975. The relationship between the concentrations of tryptophan and 5-hydroxyindoleacetic acid in rat brain and cerebrospinal fluid. J. Neurochem. 25:3551-3552.

15. Goldman, R., and F. Naider. 1974. Permeation and stereospecificity of hydrolysis of peptide esters with intact lysosomes in vitro. Biochim. Biophys. Acta. 338:224-233.

16. Reeves, J. P. 1979. Accumulation of amino acids by lysosomes incubated with amino acid methyl esters. J. Biol. Chem. 254:89148921.

17. Gahl, W. A., N. Bashan, F. Teitze, I. Bernardini, and J. D. Schulman. 1982. Cystine transport is defective in isolated leukocyte lysosomes from patients with cystinosis. Science (Wash. DC). 217:1263-1265.

18. Jonas, A. J., M. L. Smith, and J. A. Schneider. 1982. ATP 
dependent lysosomal cystine efflux is defective in cystinosis. J. Biol. Chem. 257:13185-13188.

19. Gillman, P. K., J. R. Bartlett, P. K. Bridges, B. D. Kantamaneni, and G. Curzon. 1980. Relationships between tryptophan concentrations in human plasma, cerebrospinal fluid and cerebral cortex following tryptophan infusion. Neuropharmacology. 19:1241-1242.

20. Denckla, W. D., and H. K. Dewey. 1967. The determination of tryptophan in plasma, liver, and urine. J. Lab. Clin. Med. 69:160-168.

21. Lee, P. L. Y. 1974. Single-column system for accelerated amino acid analysis of physiological fluids using five lithium buffers. Biochem. Med. 10:107-121.

22. Sharlit, H. 1932. A method for the quantitative estimation of indoxyl compounds in urine. J. Biol. Chem. 99:537-545.

23. Seifert, W. E., Jr., J. L. Foxx, and I. J. Butler. 1980. Age effect on dopamine and serotonin metabolite levels in cerebrospinal fluid. Ann. Neurol. 8:38-42.
24. Zerez, C. R., S. J. Lee, and K. R. Tanka. 1987. Spectrophotometric determination of oxidized and reduced pyridine nucleotides in erythrocytes using a single extraction procedure. Anal. Biochem. 164:367-373.

25. Poklis, A., and M. A. Mackell. 1982. Evaluation of a modified alcohol dehydrogenase assay for the determination of ethanol in blood. Clin. Chem. 28:2125-2127.

26. Jonas, A. J. 1986. Cystine transport in purified rat liver lysosomes. Biochem. J. 236:671-677.

27. Bradford, M. M. 1976. A rapid and sensitive method for the quantitation of microgram quantities of protein utilizing the principle of protein-dye binding. Anal. Biochem. 72:248-254.

28. Greenwood, M. H., J. Friedel, A. J. Bond, G. Curzon, and M. H. Lader. 1974. The acute effects of intravenous infusion of $L-$ tryptophan in normal subjects. Clin. Pharmacol. Ther. 16:455-464. 